\title{
Synaptic transmission of graded membrane potential changes and spikes between identified visual interneurons
}

\author{
Diana Rien, Roland Kern and Rafael Kurtz \\ Department of Neurobiology, Faculty of Biology, Bielefeld University, Postfach 1001 31, 33501 Bielefeld, Germany
}

Keywords: fly, synaptic transmission, visual system, voltage clamp

\begin{abstract}
Several physiological mechanisms allow sensory information to be propagated in neuronal networks. According to the conventional view of signal processing, graded changes of membrane potential at the dendrite are converted into a sequence of spikes. However, in many sensory receptors and several types of mostly invertebrate neurons, graded potential changes have a direct impact on the cells' output signals. The visual system of the blowfly Calliphora vicina is a good model system to study synaptic transmission in vivo during sensory stimulation. We recorded extracellularly from an identified motion-sensitive neuron while simultaneously measuring and controlling the membrane potential of individual elements of its presynaptic input ensemble. The membrane potential in the terminals of the presynaptic neuron is composed of two components, graded membrane potential changes and action potentials. To dissociate the roles of action potentials and graded potential changes in synaptic transmission we used voltage-clamp-controlled current-clamp techniques to suppress the graded membrane potential changes without affecting action potentials. Our results indicate that both the graded potential and the action potentials of the presynaptic neuron have an impact on the spiking characteristics of the postsynaptic neuron. Although a tight temporal coupling between pre- and postsynaptic spikes exists, the timing between these spikes is also affected by graded potential changes. We propose that the control of synaptic transfer of a dynamically complex signal by graded changes in membrane potential and spikes is useful to enable a temporally precise coupling of spikes in response to sudden transitions in stimulus intensity.
\end{abstract}

\section{Introduction}

Analog signalling in neurons has traditionally been believed to be limited to the somato-dendritic domain in most brain regions, whereas information transmission to the postsynaptic neuron requires conversion of this graded information into trains of action potentials. However, certain types of neurons in invertebrates have long been known to use combinations of graded potential changes and action potentials in synaptic information transfer (for reviews see Hooper \& DiCaprio, 2004; Marder \& Calabrese, 1996; Selverston et al., 1976). Recent research revealed that also in mammalian cortex and brainstem analog signalling plays a significant role in information transmission (Alle \& Geiger, 2006; Awatramani et al., 2005; Shu et al., 2006; for reviews see Juusola et al., 1996; Alle \& Geiger, 2008).

In the fly peripheral visual system, the rate of information transfer at graded synapses was concluded to be significantly higher than at synapses using presynaptic spike train coding (de Ruyter van Steveninck \& Laughlin, 1996). The axonal propagation of combined graded and spike signals reaches even higher information rates than either of them alone (Haag \& Borst, 1998). Thus, the synaptic transfer capacity might benefit from such a mixed presynaptic signal, if both graded potential changes and action potentials were to contribute to the signal transmission.

Correspondence: Dr D. Rien, as above.

E-mail: drien@uni-bielefeld.de

Received 25 January 2011, revised 23 May 2011, accepted 15 June 2011
The visual system of the blowfly Calliphora vicina is an ideal experimental model system to study synaptic transmission, because dual recordings of individually identified, pre- and postsynaptic neurons can be performed in nearly intact, non-anaesthetized animals during sensory stimulation. The neurons analysed in the present study, neurons of the vertical system (VS neurons), belong to the motionsensitive tangential neurons, which integrate information about retinal image shifts caused, for example, by the fly's ego motion (for reviews, see Egelhaaf et al., 2002; Borst et al., 2010). VS neurons integrate the outputs of many local, retinotopically organized motion-sensitive elements (Hengstenberg et al., 1982; Krapp et al., 1998). In their axons, prominent graded potential fluctuations are superimposed with action potentials of variable amplitude. One of their postsynaptic targets, the V1 neuron, receives input from VS neurons and converts this input into sequences of spikes, which are propagated to the contralateral brain hemisphere (Kurtz et al., 2001). The overall synaptic transfer between VS neurons and V1, which is most likely mediated by chemical as well as electrical contact synapses (Kalb et al., 2006; Haag \& Borst, 2008; Beckers et al., 2009), was shown to operate almost linearly over a wide range of presynaptic potential fluctuations (Kurtz et al., 2001; Warzecha et al., 2003). Additionally, a tight temporal coupling between pre- and postsynaptic spikes was observed (Warzecha et al., 2003; Haag \& Borst, 2008; Beckers et al., 2009). However, until now it has been not possible to separate presynaptic spikes and graded potential components during visual stimulation and to study their respective roles in synaptic transmission. 
In the present study, we applied a recently developed method, called voltage-clamp-controlled current clamp (VCcCC), which permits intracellular recordings of transient membrane potential changes along with compensation of slow membrane potential changes (Sutor et al., 2003). This technique allows us to control the graded potential changes while preserving the spike patterns elicited by sensory stimulation. Using this approach we discriminate between the effects of spikes and graded potential changes of presynaptic VS neurons on the postsynaptic V1 neuron.

\section{Experimental procedures}

\section{Animal preparation}

All experiments were performed on approximately 3-day-old female blowflies (Calliphora vicina), bred in the Neurobiology Department, Bielefeld University. The animal preparation has been described in detail elsewhere (Beckers et al., 2007). In short, the flies were briefly anaesthetized with $\mathrm{CO}_{2}$ and mounted ventral side up on a glass support. Their legs were cut off and their wings and abdomen were immobilized with beeswax. The proboscis was stretched out and waxed to the thorax. The head capsule was opened from behind. The air sacs and trachea, which normally cover the lobula plate, the caudal part of the third visual neuropil, were removed. All wounds were sealed with beeswax.

\section{Electrophysiology and data acquisition}

Fly V1 neurons were recorded extracellularly in their output arborization in the left hemisphere using borosilicate glass electrodes (GC150TF-10; Clark Electromedical, Edenbridge, UK) with an outer diameter of $1.5 \mathrm{~mm}$. Electrodes were filled with $2 \mathrm{M}$ potassium acetate resulting in a resistance of $1-5 \mathrm{M} \Omega$. V1 was identified by its sensitivity to downward motion in the frontal to frontolateral part of the visual field contralateral to the recording site (Hausen, 1984; Krapp et al., 2001; Kurtz et al., 2001). The signal was amplified using an npi (npi electronics, Tamm, Germany) SEC-10 amplifier with a SEC-EXT head stage attached.

VS neurons were recorded intracellularly in the right hemisphere. Sharp borosilicate electrodes were used to penetrate the neurons in their axons close to their output region. Electrodes (borosilicate GC100TF-10; Clark Electromedical; $1 \mathrm{~mm}$ outer diameter) were pulled using a Flaming/Brown puller (P97; Sutter Instruments, San Rafael, CA, USA) and filled with $2 \mathrm{~m}$ potassium acetate. Resistances were between 15 and $30 \mathrm{M} \Omega$. Another SEC-10 amplifier with the standard head stage for intracellular recordings (npi electronics) was used throughout the experiments. VS neurons were identified by their graded depolarizations during presentation of downward motion in their specific receptive field in bridged recording mode (Krapp et al., 1998). Due to their very similar receptive fields we did not distinguish between VS2 and VS3. For voltage clamp recordings we used the discontinuous single-electrode voltage-clamp technique (duty cycle $1 / 4$, switching frequency about $40 \mathrm{kHz}$ ). The high switching frequency of the amplifier allowed us to filter out artefacts in the extracellular recording resulting from cross-talk with the intracellular recording. The waveform of the amplifier's discontinuous raw output signal was examined during each recording to adjust the amplifier's filtering and gain properties to the properties of the electrode. Specific attention was given to electrode time constants being sufficiently small. During the amplifier's voltage sampling intervals the voltage drop across the electrode when injecting current pulses had to be completely decayed to a steady-state value that was exclusively determined by the neuron's membrane properties. Recordings in the VCcCC mode were performed according to Sutor et al. (2003) using the amplifier's VCcCC addendum. A discontinuous single-electrode current- and voltageclamp amplifier was modified to compensate for the slow membrane potential changes without affecting the faster ones. Low-pass filters with selectable time constants were incorporated into the voltage-clamp feedback loop to reduce the amplifier's response speed (for details see Sutor et al., 2003). This makes it possible to set the VS neuron's membrane potential to any command potential without altering VS spikes. VS spikes have previously been termed 'spikelets' in some studies to point out that the fast depolarizing transients riding on top of the graded membrane potential vary in their amplitude (Hengstenberg, 1977). In each experiment, synaptic coupling between V1 and the impaled VS neuron was confirmed by injecting small currents into the VS neuron and simultaneously monitoring the V1 response.

Extracellular potentials and intracellular potential and current traces were recorded at sampling rates of $100 \mathrm{kHz}$ by a standard $\mathrm{PC}$ with a multifunction I/O card (PD2-MFS-4_500/14; United Electronic Industries, Canton, MA, USA).

\section{Visual stimulation}

For visual stimulation we used drifting square-wave gratings generated with a custom built LED matrix consisting of $48 \times 48$ round ( $3 \mathrm{~mm}$ diameter) ultra bright blue LEDs (for details see Beckers et al., 2009). The LED matrix covered the fly's visual field from -10 to $70^{\circ}$ along the azimuth and from -45 to $25^{\circ}$ along the elevation axis (with $0^{\circ} / 0^{\circ}$ corresponding to the frontal midline of the fly). The spatial resolution of the LED matrix ranged from $1.5^{\circ}$ in the centre of the matrix to $1^{\circ}$ towards the edges. The gratings had a spatial wavelength of $25^{\circ}$ and moved with a temporal frequency of 3.125 cycles/s. Resulting from the limited spatial resolution of the LED matrix, motion of the grating is not a smooth process but a sequence of discrete steps, consisting of switching on one LED row at the leading edge of the grating and switching off one row at the trailing edge. At the given temporal frequency and wavelength settings these steps occur approximately every $20 \mathrm{~ms}$. Visual stimuli were controlled by self-written software (Visual C++ 6.0; Microsoft, Redmond, WA, USA) using the same PC and I/O card as for data acquisition.

The stimulation protocol consisted of presentation of the stationary grating for $2 \mathrm{~s}$, followed by motion of this grating in the preferred direction of the VS neuron for $4 \mathrm{~s}$, and an additional $4 \mathrm{~s}$ stationary period.

\section{Data analysis}

All data were evaluated offline using custom analysis routines written in MatLab (The MathWorks, Natick, MA, USA). V1 spikes were detected offline by thresholding the extracellularly recorded potentials (Beckers et al., 2009). For the detection of VS spikes the variance of the membrane potential within $1 \mathrm{~ms}$ time bins was calculated and an appropriate threshold was set to the variance trace. Spike detection was suspended in a $2.5 \mathrm{~ms}$ time window after each spike to avoid multiple detection of the same spike. The time of threshold crossing was taken as the onset of spiking. The amplitude of VS spikes was determined by calculating the difference between the maximum and the minimum in a $5.5 \mathrm{~ms}$ time window starting $2 \mathrm{~ms}$ before the detected VS spike. The width of VS spikes was calculated at the half maximum of the amplitude (Fig. 1).

The timing of VS-V1 spike pairs was determined in a $6 \mathrm{~ms}$ time window starting $1 \mathrm{~ms}$ before the detection of the VS spike. The timing 


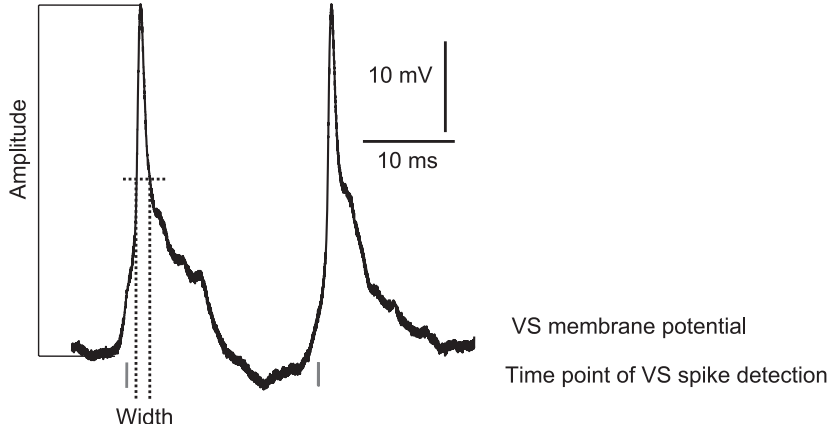

FIG. 1. Parameters of VS spikes. The amplitude of VS spikes was determined by calculating the difference between the maximum and the minimum value in a 5.5-ms time window starting $2 \mathrm{~ms}$ before the time point at which a VS spike was detected (grey vertical bars) by the procedure described in the Methods. The width of VS spikes was calculated at half the maximum amplitude.

was calculated as the time difference between the onset of a VS spike and the immediately following V1 spike.

Probabilities of binomial distributions and the corresponding 95\% confidence intervals were calculated according to the adjusted Wald method (Wilson, 1927; Agresti \& Coull, 1998).

To test for significant differences between the conditions investigated a Student's $t$-test was applied, considering a significance level of $P<0.05$. In the case of only few samples/data points we applied the Mann-Whitney $U$-test considering a $P<0.05$ significance level. The data were analysed for normal distribution by applying the singlesample Kolmogorov-Smirnov goodness-of-fit hypothesis test. The correlation was calculated according to Pearson. Unless otherwise stated, all values are expressed as means \pm SEM; $n$ refers to the number of stimulus repetitions per neuron pair.

\section{Results}

We investigated synaptic transmission between identified motionsensitive neurons in the third visual neuropil of the fly brain. The presynaptic signals at the synapses under study consist of both graded and spike-like changes in membrane potential. The major goal of our study was to clarify the interaction of these two signal components in the presynaptic neuron and their roles in controlling the postsynaptic neuron's spiking activity.

In vivo recordings were performed from the V1 neuron and simultaneously from one of its presynaptic neurons (VS1-VS3). Spike trains of the V1 neuron were recorded extracellularly in its dendritic output arborization, whereas the membrane potential of one of the VS neurons was recorded intracellularly from its axon close to its axon terminals. We recorded from nine VS-V1 neuron pairs. Based on the response to vertical and horizontal motion presented in various regions of the visual field and the known receptive field properties of the VS neurons (Krapp et al., 1998) three of the recorded neuron pairs were classified as VS1-V1 and six as VS2/3-V1. Due to their very similar receptive fields and response properties we did not distinguish between VS2 and VS3.

VS neurons respond to visual motion in their preferred direction (downward) with a graded depolarization superimposed with spikes (Fig. 2A). The graded shift in membrane potential shows average amplitudes of $10-15 \mathrm{mV}$. VS spikes are very variable in amplitude, up to a maximum depolarization of approximately $40 \mathrm{mV}$. Motion in the opposite direction leads to a hyperpolarization of up to $-10 \mathrm{mV}$ relative to the resting membrane potential.

V1 receives strong excitatory input from distal VS1-VS3 neurons and weaker, most likely indirect input from more proximal VS neurons (Kurtz et al., 2001; Warzecha et al., 2003; Kalb et al., 2006; Haag \& Borst, 2008) via electrical coupling with their neighbors
A

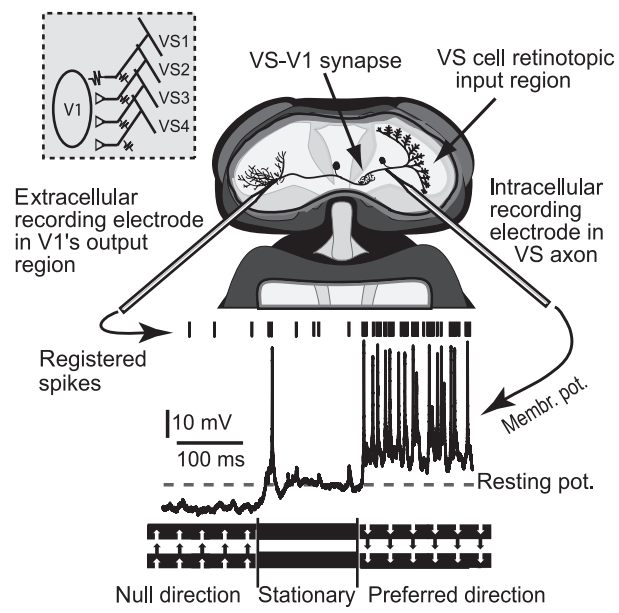

$\mathbf{B}$
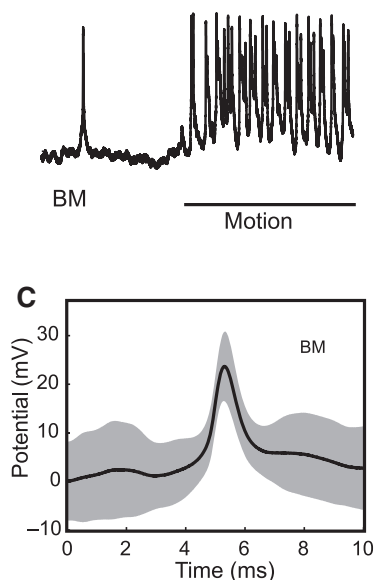
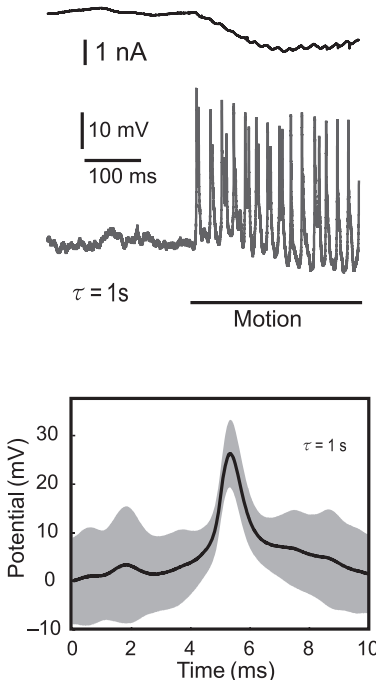
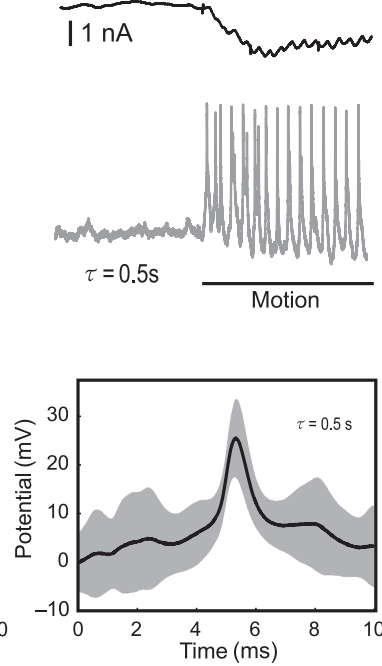

FIG. 2. (A) Experimental set-up used for dual recordings of one VS neuron and V1 during visual motion stimulation. For synaptic coupling between VS neurons and the V1 neuron in the fly motion pathway see inset. The signal is conducted to the axon terminal of VS where it is transferred to V1, which branches in the contralateral brain hemisphere. V1 is thought to receive direct input from three or four out of 10 VS neurons (Kurtz et al., 2001; Warzecha et al., 2003; Kalb et al., 2006; Haag \& Borst, 2008), which are electrically coupled with their respective neighbours (Haag \& Borst, 2004). Note that the exact nature of synapses between individual VS neurons and V1, being electrical, chemical or mixed, is controversial (Haag \& Borst, 2008; Beckers et al., 2009). VS neurons were impaled with intracellular electrodes close to the neuron's axon terminals to ensure that their presynaptic membrane potential was properly controlled by the voltage clamp command potential. V1 was recorded extracellularly at the contralateral output region. During constant-velocity motion in the non-preferred direction (upwards) VS neurons respond with graded hyperpolarizations. Motion stimulation in the preferred direction (downward) results in graded depolarization superimposed with spikes of variable amplitude. (B) Effect of voltage-clamp-controlled current clamp (VCcCC) on membrane potential modulations. Sample traces of intracellular recordings from VS1 in bridged mode (BM, left) and VCcCC with amplifier time constants of $\tau=1 \mathrm{~s}$ (middle) and $\tau=0.5 \mathrm{~s}$ (right). The bottom traces display the membrane potential fluctuations; the upper traces show the time course of current injected during VCcCC. (C) Averaged action potentials of VS1 in response to the stationary pattern for the same recording conditions as indicated above. The grey shaded area displays the standard deviation. Panel A was redrawn from Beckers et al. (2009). 
(Haag \& Borst, 2004). Although it is clear that electrical connections play a role in transmission between VS and V1, it is not yet clear which of the VS neurons express (additionally or exclusively) chemical synaptic contacts to V1 (Haag \& Borst, 2008; Beckers et al., 2009). Despite the fact that VS-to-V1 contact is formed at least partially via electrical synapses, making synaptic transfer bidirectional (Haag \& Borst, 2008; Beckers et al., 2009), we refer to VS as the presynaptic neuron and to V1 as postsynaptic. These notations are useful with regard to the direction of the major signal flow, because VS neurons receive, in contrast to V1, direct input from local motionsensitive elements upstream in the visual pathway.

In V1, postsynaptic signals from its small dendrite are converted into spikes and conveyed via a long axon to a large output arborization in the contralateral lobula plate. V1's spike rate increases to $250 \mathrm{~Hz}$ when its presynaptic elements are stimulated by motion in its preferred direction. Without motion V1 shows a spontaneous activity of about 10-30 Hz (Kurtz et al., 2001; Figs 2 and 4).

\section{Suppression of graded presynaptic membrane potential changes by voltage-clamp controlled current clamp}

To investigate whether graded changes in membrane potential and spiking activity of the presynaptic neuron affect synaptic transmission in a distinct way, we adapted the $\mathrm{VCcCC}$ technique from Sutor et al. (2003). This technique allowed us to control, during sensory stimulation, the slow, graded changes in membrane potential without significant attenuation of the fast spikes. Synaptic transmission of the graded presynaptic membrane potential alone, in the absence of spikes, has already been described using normal voltage-clamp (Beckers et al., 2007). By holding the presynaptic membrane potential at a preset depolarization level, it was shown that graded depolarization effectively induced spiking in the postsynaptic neuron.

Our intracellular recordings from VS neurons confirmed that $\mathrm{VCcCC}$ is a suitable method to separate the spikes from the graded change in membrane potential. We clamped the membrane potential during visual stimulation to its overall resting level, and allowed only fast changes in membrane potential, including in particular the typical spikes of variable amplitude, to be propagated along the axon (Fig. 2B). We adapted $\mathrm{VCcCC}$ to our requirements by determining the adequate time constant of the amplifier circuit (Sutor et al., 2003). The VCcCC time constant is critical to separate the range of slow membrane potential modulations that is affected by the voltage clamp from the range of fast modulations that is left unaffected. Figure 2B shows sample traces of the amplifier's operation. After impalement of one VS neuron, its mean resting potential was determined and the neuron was then clamped to this value $(-40 \mathrm{mV})$ during visual motion stimulation. Under control conditions in the bridged mode (BM) of the amplifier, grating motion elicited a graded depolarization of the VS neuron's membrane potential with spikes superimposed (Fig. 2B, left). After selecting a time constant of $\tau=1 \mathrm{~s}$ and switching to the VCcCC mode, the same stimulus produced a response with only a small residual graded component (Fig. 2B, middle), which is most prominent during the first $100 \mathrm{~ms}$ after motion onset. The injected clamp current counteracting the neuron's slow potential changes showed, in contrast to normal voltage clamping (Beckers et al., 2009), no distinct fast current transients (Fig. 2B, middle, upper trace). A further decrease of the VCcCC time constant to $\tau=0.5 \mathrm{~s}$ led to more complete suppression of the graded potential component, reaching the steady state earlier after the onset of motion (Fig. 2B, right). Importantly, no pronounced current transients were found in the injected compensation current, indicating that a time constant of $\tau=0.5 \mathrm{~s}$ is sufficiently small to allow suppression of the graded potential changes but not too small to lead to attenuation of VS spikes (Fig. 2B and C). Even though we used much shorter time constants of the VCcCC than in the original study (Sutor et al., 2003) the width of VS spikes was not significantly different between BM and VCcCC (Fig. 2C). The amplitude of VS spikes was even slightly enhanced in the $\mathrm{VCcCC}$ mode compared with BM, contrary to what would be expected if spikes were attenuated by voltage-clamping the neuron in the VCcCC mode. This effect is considered in more detail further below. When a visual motion stimulus in the neurons preferred direction was presented, VS spikes often ride on top of fast depolarizations (Fig. 2B), resembling excitatory postsynaptic potentials. These depolarizations occasionally occurred phase-locked to the advance of the grating by a LED row and are likely to contribute to spike generation. However, this phase-locking does not affect any of the conclusions of this study. As indicated by the presence of corresponding modulations in the amplifier current, these depolarizations are slightly suppressed during voltage-clamping in $\mathrm{VCcCC}$ at a time constant of $\tau=0.5 \mathrm{~s}$. Voltage-clamping in VCcCC at a time constant of $\tau=0.5 \mathrm{~s}$ also suppressed hyperpolarization during motion in the neuron's null direction (data not shown).

To evaluate the amplifier's operation, we analysed the frequency distribution of the VS membrane potential changes during visual stimulation. To this end, we compared frequency power spectra of the VS membrane potential under control conditions and VCcCC (Fig. 3A). The occurrence of spikes is reflected in the power within the high-frequency range. In this range, a high degree of similarity was present between the control condition, i.e. in the amplifier's BM, and VCcCC (Table 1; Fig. 3). In contrast to the high frequencies, the
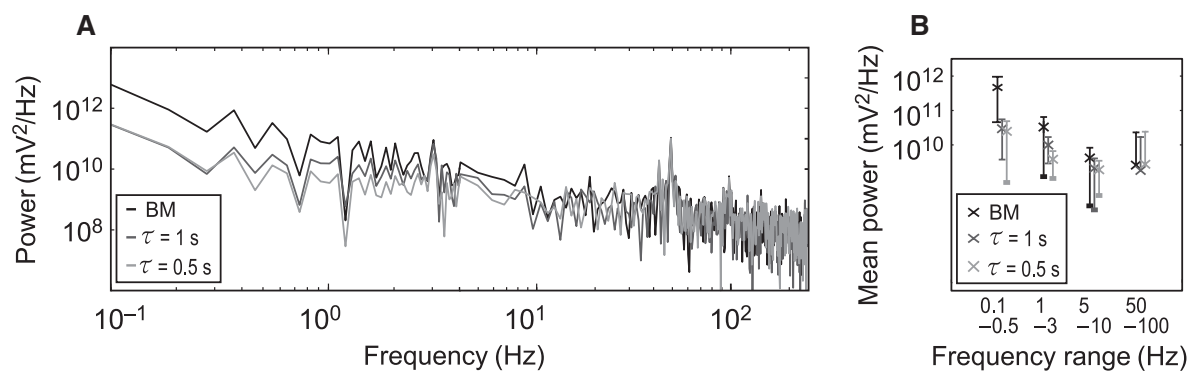

FIG. 3. (A) Frequency power spectrum of membrane potential modulations during the stimulation sequence. Fourier transformations of the membrane potential for $\mathrm{BM}$ and VCcCC are compared. The prominent peaks at slightly below $50 \mathrm{~Hz}$ are not present during stationary pattern presentation (data not shown). Thus, these peaks originate from the phase-locking of depolarizations to the advance of the grating by single LED rows, which occurs approximately every 20 ms for the motion stimulus used. (B) Mean power of frequencies in the range of 50-100, 5-10, 1-3 and 0.1-0.5 Hz; error bars indicate standard deviations. For the range of 50-100 Hz no negative values are plotted due to the logarithmic scaling of the graph. 
TABLE 1. Mean power of frequencies in different frequency ranges calculated from the frequency power spectrum of membrane potential modulations during motion stimulation as displayed in Fig. 3

\begin{tabular}{lcrr}
\hline & \multicolumn{2}{c}{ Mean power $\pm \mathrm{SD}\left(\mathrm{mV}^{2} / \mathrm{Hz}\right)$} & \\
\cline { 2 - 4 } Frequency range $(\mathrm{Hz})$ & Bridged mode & $\tau=1 \mathrm{~s}$ & $\tau=0.5 \mathrm{~s}$ \\
\hline $50-100$ & $2.56 \times 10^{9} \pm 2.05 \times 10^{10}$ & $1.81 \times 10^{9} \pm 1.51 \times 10^{10}$ & $2.73 \times 10^{9} \pm 2.13 \times 10^{10}$ \\
$5-10$ & $4.19 \times 10^{9} \pm 4.02 \times 10^{9}$ & $2.09 \times 10^{9} \pm 1.96 \times 10^{9}$ & $1.89 \times 10^{9} \pm 1.55 \times 10^{9}$ \\
$1-3$ & $3.3 \times 10^{10} \pm 3.18 \times 10^{10}$ & $9.91 \times 10^{9} \pm 7.01 \times 10^{9}$ & $3.83 \times 10^{9} \pm 2.77 \times 10^{9}$ \\
$0.1-0.5$ & $4.97 \times 10^{11} \pm 4.51 \times 10^{11}$ & $2.94 \times 10^{10} \pm 2.57 \times 10^{10}$ & $2.48 \times 10^{10} \pm 2.4 \times 10^{10}$ \\
\hline
\end{tabular}

power in the low-frequency regime, which is characteristic of the graded change in membrane potential, declined considerably in $\mathrm{VCcCC}$ compared with the control. Note that even when the visual stimulus is moving at a constant velocity the membrane potential of VS neurons measured in BM is expected to exhibit slow modulations. These modulations result from the temporal dynamics of correlationtype motion detectors (Borst \& Egelhaaf, 1989), the non-random spatial structure of the grating pattern and activity fluctuations which do not depend on any visual input (Longden \& Krapp, 2009; Maimon et al., 2010; Rosner et al., 2010). No prominent differences were observed between the different time constants of VCcCC (Fig. 3A and B). Therefore, we set the amplifier to $\tau=0.5 \mathrm{~s}$, because, as is evident from Fig. 2B, this time constant is more effective than $\tau=1 \mathrm{~s}$ in suppressing the graded potential component instantaneously after motion onset.

\section{Presynaptic spikes affect postsynaptic activity even when sustained presynaptic graded potential changes are blocked}

Our major goal was to address the question of how the suppression of the presynaptic VS neuron's graded potential changes in response to visual stimulation is reflected in the postsynaptic spiking rate. Prior to investigating synaptic transmission between VS and V1, we examined the influence of $\mathrm{VCcCC}$ on the rate of VS spikes. When clamping VS in the $\mathrm{VCcCC}$ mode to its resting membrane potential the pattern of spiking of the VS neuron was largely preserved (Fig. 2B). In Fig. 4 the spike rates observed in one VS1-V1 dual recording (Fig. 4A and B) and in one VS2/3-V1 dual recording (Fig. 4C and D) are plotted. The neuron pairs with the largest overall number of stimulus repetitions were chosen for this illustration. All investigated VS-V1 neuron pairs showed the same tendencies in their response characteristics, although with lower numbers of stimulus repetitions. The spike rates of the VS neurons remained unchanged by $\mathrm{VCcCC}$ when the grating was stationary (median spike rate for VS1: BM: $1 \mathrm{~Hz}, n=12$; VCcCC: $1 \mathrm{~Hz}, n=8$; Mann-Whitney $U$-test, $P=0.258$; for $\mathrm{VS} 2 / 3$ : BM: $0 \mathrm{~Hz}, n=17$; VCcCC: $0 \mathrm{~Hz}, n=3$; Mann-Whitney $U$-test, $P=0.633$; see Fig. $4 \mathrm{~A}$ and $\mathrm{C}$, left).

In contrast, both in VS1 and in VS2/3, during motion stimulation the rate of spikes was slightly reduced with $\mathrm{VCcCC}$ compared with BM (median spike rate for VS1: BM: $72 \mathrm{~Hz}, n=12$; VCcCC: $51 \mathrm{~Hz}$, $n=8$; Mann-Whitney $U$-test, $P=0.010$; for VS2/3: BM: $40 \mathrm{~Hz}$, $n=17$; VCcCC: $25 \mathrm{~Hz}, n=3$; Mann-Whitney $U$-test, $P=0.026$; see Fig. $4 \mathrm{~A}$ and $\mathrm{C}$, right). The persistence of spiking while applying $\mathrm{VCcCC}$ to the resting membrane potential level shows that spike
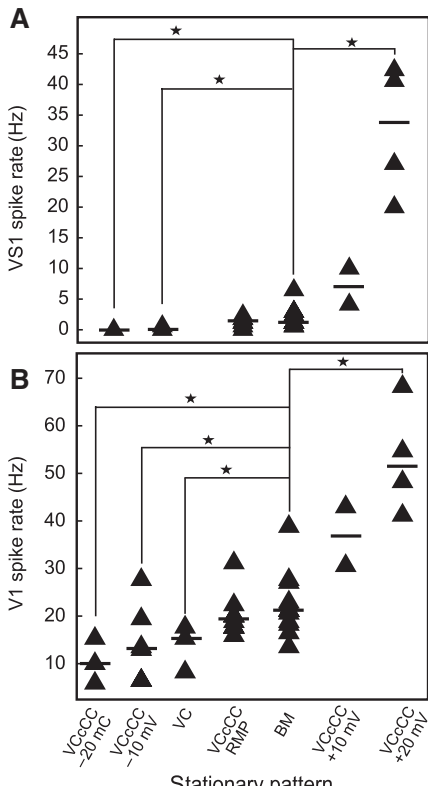

Stationary pattern
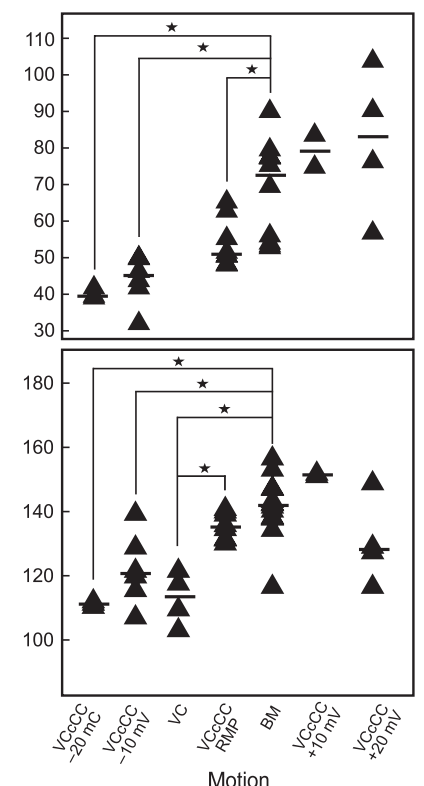

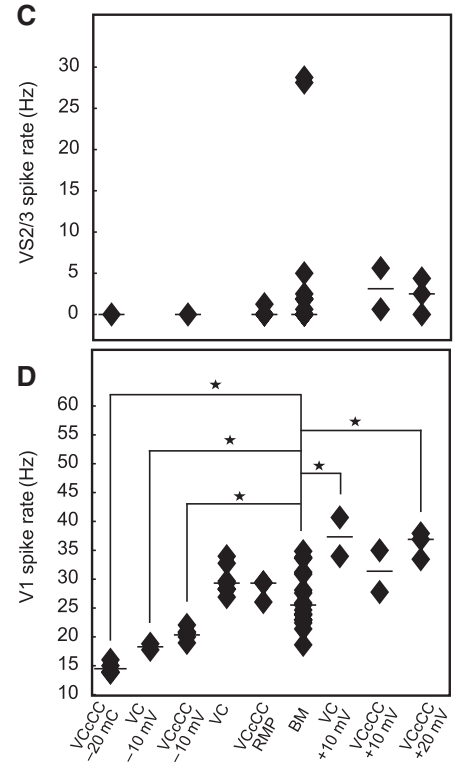

Stationary pattern

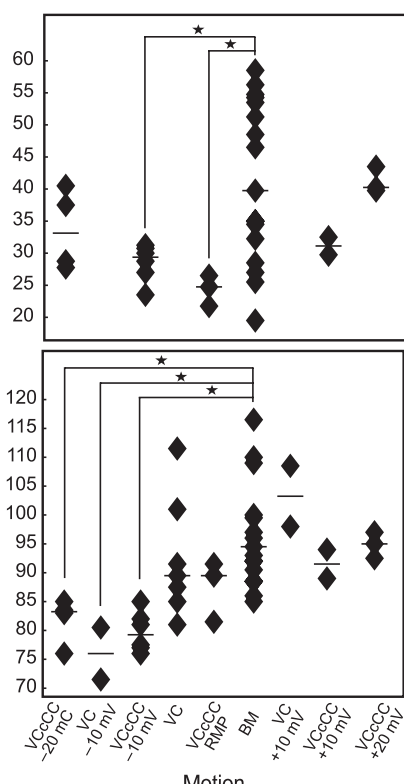

Motion

FIG. 4. (A) Spike rates of VS1 in response to the stationary pattern (left) and to downward motion (right). The horizontal line represents the median of the respective trials for each stimulus condition. Every trial consists of $2 \mathrm{~s}$ of stationary pattern presentation and $4 \mathrm{~s}$ of pattern motion. (B) Spike rates of V1 in response to the stationary pattern (left) and to downward motion (right). The asterisk indicates statistical significance at $P<0.05$, Mann-Whitney $U$-test. Note that the difference between bridged mode and VCcCC during stimulus motion was just not significant, $P=0.053$. (C) Spike rates of VS2/3. (D) Spike rates of V1. In C and D the style of the data presentation is the same as in $\mathrm{A}$ and $\mathrm{B}$, respectively. 
generation in VS does not critically depend on sustained depolarization close to the axon terminals. There are two plausible reasons for this finding which are not mutually exclusive. First, spikes might be effectively elicited by the transient depolarizations, which remain present during VCcCC. Second, the site of spike generation might be in a large electrotonic distance from the axonal recording site, presumably close to or in the dendrite of the VS neuron. Regardless of the reason, the fact that recordings under $\mathrm{VCcCC}$ conditions do not impact on the spike generation mechanism is beneficial for our intention to block only the sustained graded depolarization but leave spikes largely unaffected.

During presentation of a stationary pattern the spike rate of the postsynaptic V1 neuron did not change when the presynaptic VS1 neurons was clamped in the $\mathrm{VCcCC}$ mode, but decreased slightly under full voltage clamp of the VS1 neuron (median spike rate for V1: $\mathrm{BM}: 22 \mathrm{~Hz}, n=12$; VCcCC: $20 \mathrm{~Hz}, n=8$; VC: $16 \mathrm{~Hz}, n=4$; Mann-Whitney $U$-test: BM vs. VCcCC: $P=0.254$; $\mathrm{BM}$ vs. VC: $P=0.008$; see Fig. 4B, left). Voltage clamping the $\mathrm{VS} 2 / 3$ neuron during the presentation of the stationary pattern did not change the spike rate of V1 (median spike rate V1: BM: $26 \mathrm{~Hz}, n=17$; VCcCC: $29 \mathrm{~Hz}, n=3$; VC: $29 \mathrm{~Hz}, n=7$; Mann-Whitney $U$-test: BM vs. VCcCC: $P=0.833$; BM vs. VC: $P=0.869$; Fig. $4 \mathrm{D}$, left).

Beckers et al. (2007) demonstrated a reduction of spike rate of V1 when the membrane potential of one of the presynaptic VS neurons was clamped to its resting level during motion in the preferred direction. In the present study, we compared the effects of full voltage clamp as used in the previous study with those of $\mathrm{VCcCC}$ to determine whether presynaptic spikes alone, in the absence of a sustained graded component, affect postsynaptic spiking. During motion in the preferred direction the firing rate of $\mathrm{V} 1$ was slightly but not statistically not significantly reduced relative to control (BM) when the graded potentials of the presynaptic VS1 or VS2/3 neuron were clamped to resting level by VCcCC (Fig. 4B and D, right). The spike rate of the $\mathrm{V} 1$ neuron was even more reduced when the membrane potential of VS1 was fully clamped to resting level. No further reduction in spike rate was observed when VS2/3 was voltage clamped to resting level compared with voltage-clamping the neuron in the VCcCC mode (median spike rate of V1 in VS1-V1 dual recording: $\mathrm{BM}$ : $143 \mathrm{~Hz}, n=12$; VCcCC: $137 \mathrm{~Hz}, n=8$; VC: $114 \mathrm{~Hz}$, $n=4$; Mann-Whitney $U$-test: $\mathrm{BM}$ vs. VCcCC: $P=0.053$; $\mathrm{BM}$ vs. $\mathrm{VC}: P=0.031$; median spike rate of $\mathrm{V} 1$ in $\mathrm{VS} 2 / 3-\mathrm{V} 1$ dual recording: BM: $95 \mathrm{~Hz}, n=17$; VCcCC: $90 \mathrm{~Hz}, n=3$; VC: $90 \mathrm{~Hz}, n=7$; Mann-Whitney $U$-test: BM vs. VCcCC: $P=0.090$; BM vs. VC: $P=0.266$; see asterisks in Fig. $4 \mathrm{~B}$ and D). These results suggest that the postsynaptic spike rate is affected by both the spike rate and the graded potential changes of the presynaptic neuron. However, our results would also be compatible with the notion that synaptic transmission is based on presynaptic spikes alone, because the spike rates of VS and $\mathrm{V} 1$ are affected by $\mathrm{VCcCC}$ in a similar way. To be able to interpret these results, it is important to consider a previous study showing that voltage clamping VS neurons to sustained presynaptic depolarization (in the absence of spikes) increases the spike rate of V1 (Beckers et al., 2007). We therefore conclude that graded and spike signals from the presynaptic VS neuron both contribute to synaptic transmission.

\section{Effect of presynaptic graded de- or hyperpolarization on postsynaptic activity}

We tested how a graded de- or hyperpolarization of a VS neuron during voltage-clamping in the VCcCC mode affects spiking in VS and V1. Depolarization of VS1 by applying VCcCC markedly enhanced its spike rate during presentation of a stationary pattern. In contrast, the corresponding decrease in spiking when $\mathrm{VCcCC}$ is applied to induce a sustained hyperpolarization level is much less pronounced compared with control conditions, because the spontaneous spike rate of VS1 is already fairly low (median spike rate of VS1: BM: $1 \mathrm{~Hz}, n=12 ; \mathrm{VCcCC}_{+10 \mathrm{mV}}: 7 \mathrm{~Hz}, n=2 ; \mathrm{VCcCC}_{+20 \mathrm{mV}}$ : $34 \mathrm{~Hz}, n=4$; $\mathrm{VCcCC}_{-10 \mathrm{mV}}: 0 \mathrm{~Hz}, n=6$; $\mathrm{VCcCC}_{-20 \mathrm{mv}}: 0 \mathrm{~Hz}$, $n=3$; Mann-Whitney $U$-test: $\mathrm{BM}$ vs. $\mathrm{VCcCC}_{+10 \mathrm{mV}}: P=0.110$; $\mathrm{BM}$ vs. $\mathrm{VCcCC}_{+20 \mathrm{mv}}: P=0.001 ; \mathrm{BM}$ vs. $\mathrm{VCcCC}_{-10} \mathrm{mv}: P=0.001 ; \mathrm{BM}$ vs. VCcCC $-20 \mathrm{mv}: P=0.013$; see asterisks in Fig. 4A, left). However, the spike rate in response to motion is strongly attenuated compared with $\mathrm{BM}$ conditions when setting the neuron to a hyperpolarized level. Differences in spiking activity were not observed when the neuron's potential was clamped to a graded depolarized level, presumably because the motion-induced change in graded potential and the artificially sustained depolarization by $+10 \mathrm{mV}$ are similar in strength. Further artificial depolarization to $+20 \mathrm{mV}$ might cause tonic inactivation of sodium channels (median spike rate of VS1: BM: $72 \mathrm{~Hz}$, $n=12 ; \mathrm{VCcCC}_{+10 \mathrm{mV}}: 79 \mathrm{~Hz}, n=2$; $\mathrm{VCcCC}_{+20 \mathrm{mV}}: 83 \mathrm{~Hz}, n=4$; $\mathrm{VCcCC}_{-10 \mathrm{mV}}: 45 \mathrm{~Hz}, n=6$; $\mathrm{VCcCC}_{-20 \mathrm{mV}}: 40 \mathrm{~Hz}, n=3$; MannWhitney $U$-test, $\mathrm{BM}$ vs. $\mathrm{VCcCC}_{+10 \mathrm{mv}}: P=0.418$; $\mathrm{BM}$ vs. $\mathrm{VCcCC}_{+20 \mathrm{mv}}: P=0.163$; $\mathrm{BM}$ vs. $\mathrm{VCcCC}_{-10 \mathrm{mV}}: P=0.001 ; \mathrm{BM}$ vs. VCcCC $-20 \mathrm{mv}: P=0.004$; see asterisks in Fig. 4A, right).

The spike rate of the postsynaptic V1 neuron depends on the graded membrane potential level of the VS1 neuron (Fig. 4B). Hyperpolarization of VS1 by voltage-clamping in $\mathrm{VCcCC}$ led to a decrease in spiking activity of V1 compared with BM, whereas depolarization of VS1 increased the spike rate of V1. The increments in spike rate of V1 when VS1 was set to a depolarized level were less consistent when the motion stimulus was presented compared with the fly viewing the stationary pattern (median spike rate in response to the stationary pattern: BM: $22 \mathrm{~Hz}, n=12$; $\mathrm{VCcCC}_{+10 \mathrm{mV}}: 37 \mathrm{~Hz}, n=2 ; \mathrm{VCcCC}_{+20 \mathrm{mV}}: 53 \mathrm{~Hz}$, $n=4$; $\mathrm{VCcCC}_{-10 \mathrm{mV}}: 14 \mathrm{~Hz}, n=6$; $\mathrm{VCcCC}_{-20 \mathrm{mV}}: 11 \mathrm{~Hz}, n=3$; Mann-Whitney $U$-test: $\mathrm{BM}$ vs. $\mathrm{VCcCC}_{+10 \mathrm{mV}}: P=0.134$; $\mathrm{BM}$ vs. $\mathrm{VCcCC}_{+20 \mathrm{mv}}: P=0.002$; $\mathrm{BM}$ vs. $\mathrm{VCcCC}_{-10 \mathrm{mv}}: P=0.043$; BM vs. $\mathrm{VCcCC}_{-20 \mathrm{mV}}: P=0.024$; median spike rate in response to motion: BM: $143 \mathrm{~Hz}, n=12 ; \mathrm{VCcCC}_{+10 \mathrm{mV}}: 153 \mathrm{~Hz}, n=2 ; \mathrm{VCcCC}_{+20} \mathrm{mV}$ : $130 \mathrm{~Hz}, n=4$; $\mathrm{VCcCC}_{-10} \mathrm{mv}: 122 \mathrm{~Hz}, n=6$; $\mathrm{VCcCC}_{-20 \mathrm{mv}}: 114 \mathrm{~Hz}$, $n=3$; Mann-Whitney $U$-test: $\mathrm{BM}$ vs. $\mathrm{VCcCC}_{+10 \mathrm{mv}}: P=0.198$; $\mathrm{BM}$ vs. $\mathrm{VCcCC}_{+20 \mathrm{mv}}: P=0.164 ; \mathrm{BM}$ vs. $\mathrm{VCcCC}_{-10} \mathrm{mv}: P=0.003 ; \mathrm{BM}$ vs. VCcCC $-20 \mathrm{mv}: P=0.004$; see asterisks in Fig. $4 \mathrm{~B}$ left, right). This is probably due to the fact that the impact of a single presynaptic VS neuron under $\mathrm{VCcCC}$ on the response of $\mathrm{V} 1$ is masked when the concomitant inputs from the other VS neurons are strong.

VS2/3 showed the same tendencies in synaptic transmission to V1 like VS1 under the investigated conditions, although to a much lower extent (median spike rate in response to the stationary pattern: BM: $26 \mathrm{~Hz}, n=17$; $\mathrm{VCcCC}_{+10 \mathrm{mV}}: 31 \mathrm{~Hz}, n=2 ; \mathrm{VCcCC}_{+20 \mathrm{mV}}: 36 \mathrm{~Hz}$, $n=3$; $\mathrm{VCcCC}_{-10 \mathrm{mV}}: 20 \mathrm{~Hz}, n=6 ; \mathrm{VCcCC}_{-20 \mathrm{mV}}: 14 \mathrm{~Hz}, n=4$; Mann-Whitney $U$-test: $\mathrm{BM}$ vs. $\mathrm{VCcCC}_{+10 \mathrm{mv}}: P=0.187$; $\mathrm{BM}$ vs $\mathrm{VCcCC}_{+20 \mathrm{mv}}: P=0.003$; $\mathrm{BM}$ vs. $\mathrm{VCcCC}_{-10 \mathrm{mv}}: P=0.002$; $\mathrm{BM}$ vs. $\mathrm{VCcCC}_{-20 \mathrm{mV}}: P=0.003$; median spike rate in response to motion: BM: $95 \mathrm{~Hz}, n=17$; $\mathrm{VCcCC}_{+10 \mathrm{mV}}$ : $92 \mathrm{~Hz}, n=2$; $\mathrm{VCcCC}_{+20 \mathrm{mV}}$ : $95 \mathrm{~Hz}, n=3$; $\mathrm{VCcCC}_{-10 \mathrm{mV}}: 79 \mathrm{~Hz}, n=6$; $\mathrm{VCcCC}_{-20 \mathrm{mV}}: 83 \mathrm{~Hz}$, $n=4$; Mann-Whitney $U$-test: $\mathrm{BM}$ vs. $\mathrm{VCcCC}_{+10 \mathrm{mv}}: P=0.561 ; \mathrm{BM}$ vs. $\mathrm{VCcCC}_{+20 \mathrm{mv}}: P=0.957 ; \mathrm{BM}$ vs. $\mathrm{VCcCC}_{-10 \mathrm{mv}}: P=0.002 ; \mathrm{BM}$ vs. $\mathrm{VCcCC}_{-20} \mathrm{mv}: P=0.003$; see asterisks in Fig. $4 \mathrm{C}$ and $\left.\mathrm{D}\right)$. This result is consistent with the previous finding that voltage clamping VS1 affects V1's activity more than voltage clamping VS2/3 (Beckers et al., 2007).

In VS2/3 we additionally compared the effects of normal voltage clamping to a graded membrane potential with that of voltage-clamping 
in $\mathrm{VCcCC}$ at the same graded level. The postsynaptic spike rate was slightly more reduced by normal voltage clamping than by voltageclamping in $\mathrm{VCcCC}$ to the same hyperpolarized level, but the difference between the two conditions was not statistically significant (median spike rate in response to the stationary pattern: $\mathrm{BM}: 26 \mathrm{~Hz}, n=17$; $\mathrm{VCcCC}_{\mathrm{RMP}}: 29 \mathrm{~Hz}, n=3$; $\mathrm{VC}_{\mathrm{RMP}}: 29 \mathrm{~Hz}, n=7$; $\mathrm{VCcCC}_{+10 \mathrm{mV}}$ : $31 \mathrm{~Hz}, n=2$; $\mathrm{VC}_{+10 \mathrm{mV}}: 37 \mathrm{~Hz}, n=2 ; \mathrm{VCcCC}_{-10 \mathrm{mV}}: 20 \mathrm{~Hz}, n=6$; $\mathrm{VC}_{-10} \mathrm{mv}$ : $18 \mathrm{~Hz}, n=2$; Mann-Whitney $U$-test: BM vs. $\mathrm{VC}_{-10} \mathrm{mv}$ : $P=0.023$; $\mathrm{BM}$ vs. $\mathrm{VC}_{+10 \mathrm{mv}}: P=0.023$; median spike rate in response to motion: BM: $95 \mathrm{~Hz}, n=17$; VCcCC: $90 \mathrm{~Hz}, n=3$; VC: $90 \mathrm{~Hz}$, $n=7 ; \quad \mathrm{VCcCC}_{+10 \mathrm{mv}}: 92 \mathrm{~Hz}, n=2 ; \mathrm{VC}_{+10 \mathrm{mV}}: 92 \mathrm{~Hz}, n=2$; $\mathrm{VCcCC}_{-10 \mathrm{mv}}: 79 \mathrm{~Hz}, n=6$; $\mathrm{VC}_{-10} \mathrm{mv}: 76 \mathrm{~Hz}, n=2$; Mann-Whitney $U$-test: $\mathrm{BM}$ vs. $\mathrm{VC}_{-10} \mathrm{mv}: P=0.012 ; \mathrm{BM}$ vs. $\mathrm{VC}_{+10 \mathrm{mv}}$ : $P=0.339$; Fig. 4D).

\section{Shifts in graded potential of VS influence its spike amplitude and synaptic transmission to $\mathrm{V} 1$}

In mammalian cortex and hippocampus, it has been demonstrated that the efficiency of presynaptic spikes to elicit postsynaptic potentials is affected by the background membrane potential, i.e. by the presynaptic graded potential level on which a spike rides when it invades the presynaptic terminal (Alle \& Geiger, 2006; Shu et al., 2006). In one of these cases, modulations in spike shape by the graded membrane potential level were responsible for the differences in synaptic transmission (Shu et al., 2006; see also de Polavieja et al., 2005). Therefore, we analysed whether in VS neurons the graded membrane potential might play a similar role in modulating its own spiking properties and synaptic transmission to V1. In this regard, VS1 spikes elicited by visual motion were compared between bridged mode recordings and recordings under $\mathrm{VCcCC}$. We clamped the graded membrane potential of VS1 to its resting level and to various values relative to the resting level. When unmanipulated, VS1 spikes covered a large range of amplitudes, reaching on average $30 \mathrm{mV}$ (Fig. 5, middle). The spike amplitudes decreased significantly when the neurons were clamped in VCcCC to a depolarized level (Fig. 5, right). In contrast, elimination of the motion-induced graded depolarization as well as graded hyperpolarization resulted in significantly higher spike amplitudes (Fig. 5, left). These findings are compatible with previous findings showing that spike amplitude in VS neurons increases with moderate hyperpolarizing current injection (Hengstenberg, 1977). Depolarizing current injection or sodium channel blockers, on the other hand, were shown to block spiking in similar types of neurons (HS neurons; Haag et al., 1997; Haag \& Borst, 1998). A plausible explanation for these effects is the voltagedependence of sodium channel inactivation. The injection of a depolarizing current induces inactivation of voltage-gated sodium channels. We also analysed whether spike width is affected by $\mathrm{VCcCC}$, but did not find any systematic changes (data not shown).

Above we have shown that the graded membrane potential level of VS1 affects the amplitude and the rate of its own spikes as well as the rate of spikes in the postsynaptic V1 neuron. To analyse whether the graded membrane potential level also affects the timing of synaptic transmission we determined the latency between the onset of a VS1
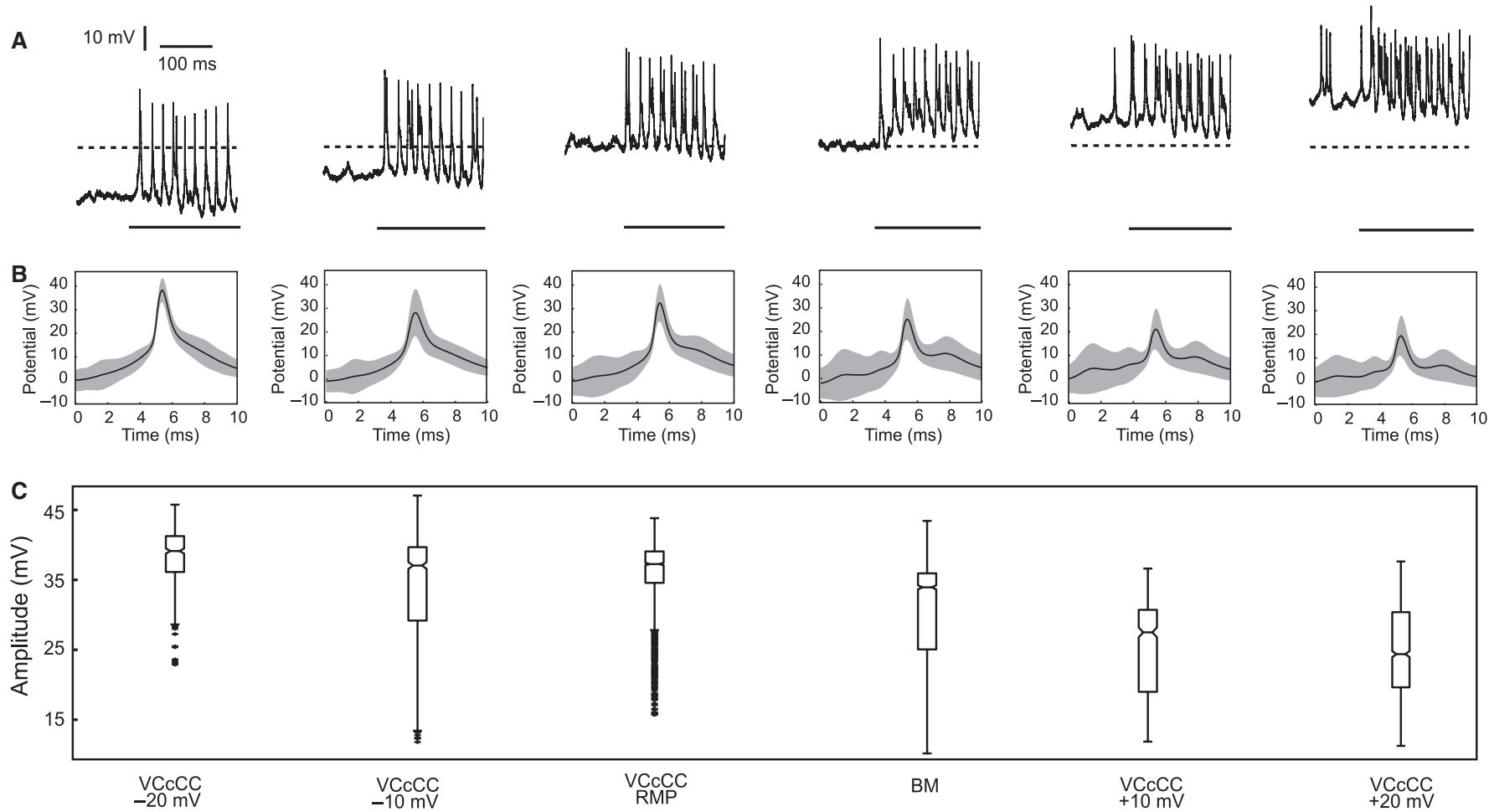

FIG. 5. (A) Presynaptic graded potential shapes amplitude of presynaptic spikes and rate of pre- and postsynaptic spikes. Upper: representative responses of a VS1 neuron to a downward drifting grating in BM and VCcCC on different graded holding potentials (see $x$-axis labels in C). The dashed line marks the resting potential $(-40 \mathrm{mV})$. The solid line indicates the motion interval. (B) VS spike average (black line). The grey shaded area displays the standard deviation. (C) Amplitude of VS1 spikes during motion stimulation under the different recording conditions. The conditions are arranged according to graded membrane potential, changing from left to right from hyperpolarized to increasingly depolarized levels. RMP, resting membrane potential. All other values are relative to RMP. Hyperpolarization results in an increase in spike amplitude; depolarization leads to a decrease in spike amplitude. Data of one representative VS1 neuron are shown (total number of VS spikes: $\mathrm{VCcCC}_{-20 \mathrm{mV}}$ : 504; $\mathrm{VCcCC}_{-10 \mathrm{mV}}: 1121$; $\mathrm{VCcCC}_{\mathrm{RMP}}$ : 1855; BM: 3534; $\mathrm{VCcCC}_{+10 \mathrm{mV}}: 701$; $\left.\mathrm{VCcCC}_{+20 \mathrm{mV}}: 1758\right)$. 

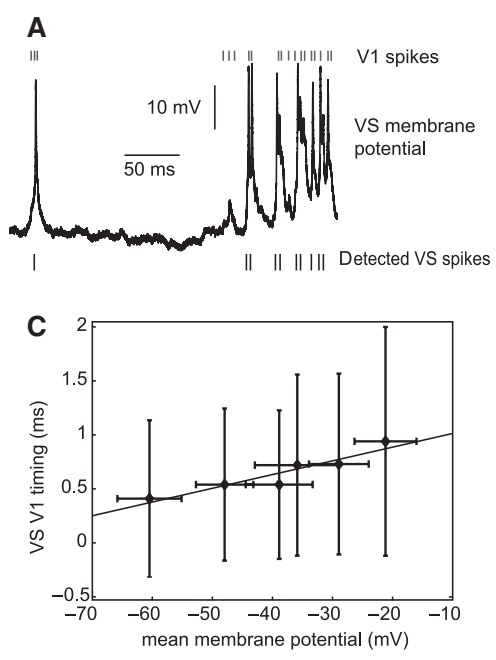
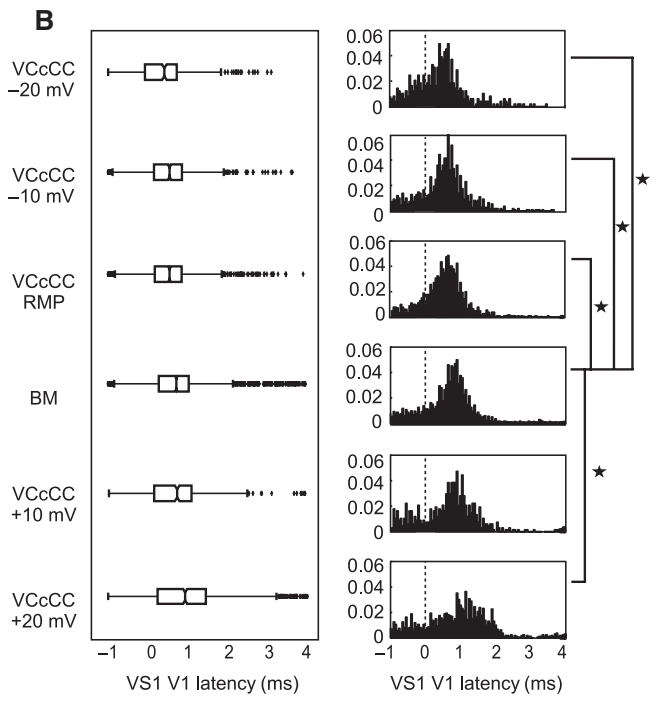

FIG. 6. Timing of pre- and postsynaptic spikes. (A) Example trace of recorded presynaptic potential fluctuations (black trace) with detected presynaptic spikes (dashes below) and simultaneously registered postsynaptic spikes (dashes on top). (B) Histograms (right) of the frequency distributions of VS1-V1 spike sequences in BM and VCcCC on different holding potentials. Data of one representative VS1-V1 neuron pair are shown (number of VS1-V1 spike pairs: VCcCC -20 mV: 504 ; $\mathrm{VCcCC}_{-10 \mathrm{mV}}$ : 1121; $\mathrm{VCcCC}_{\mathrm{RMP}}$ : 1855; BM: 3534; $\mathrm{VCcCC}_{+10 \mathrm{mV}}$ : 701; $\mathrm{VCcCC}_{+20 \mathrm{mV}}$ : 1758). The distribution was tested for normal distribution by applying the single sample Kolmogorov-Smirnov goodness-of-fit hypothesis test. To test for significant differences between the distributions a Student's $t$-test was applied, considering a significance level of $P<0.05$. The asterisk indicates statistical significance. The dashed line marks the occurrence of the VS spike; the same data are displayed in boxplots to the left. (C) Linear relationship between the mean membrane potential and the median VS1-V1 latency for the same data set $(r=0.94$, $P=0.005)$.

spike and the immediately following V1 spike (Fig. 6A). When the VS1 neuron was clamped to the resting membrane potential or to a hyperpolarized level, we observed a significant shift of the spike latency towards shorter values compared with the control condition (Fig. 6B). In contrast, the frequency distribution of the VS1V1 latencies was shifted towards higher values when the VS1 neuron was depolarized by $20 \mathrm{mV}$ in $\mathrm{VCcCC}$ (mean latencies $\pm \mathrm{SD}$ : $\mathrm{VCcCC}_{-20 \mathrm{mV}}: 0.405 \pm 0.726 \mathrm{~ms} ; \mathrm{VCcCC}_{-10 \mathrm{mv}}: 0.514 \pm 0.704 \mathrm{~ms}$ VCcCC: $0.531 \pm 0.688 \mathrm{~ms}$; BM: $0.694 \pm 0.838 \mathrm{~ms}$; $\mathrm{VCcCC}_{+10} \mathrm{mv}$ : $0.651 \pm 0.837 \mathrm{~ms}, \mathrm{VCcCC}_{+20 \mathrm{mv}}: 0.940 \pm 1.059 \mathrm{~ms}$, the asterisks indicate statistical significance, Student's $t$-test: $\mathrm{BM}$ vs. VCcCC: $P=8.7 \times 10^{-12} \mathrm{BM}$ vs. $\mathrm{VCcCC}_{-10 \mathrm{mv}}: P=8.7 \times 10^{-11}, \mathrm{BM}$ vs. $\mathrm{VCcCC}_{-20 \mathrm{mv}}: P=3.4 \times 10^{-13}, \mathrm{BM}$ vs. $\mathrm{VCcCC}_{+10 \mathrm{mV}}: P=0.22$, $\mathrm{BM}$ vs. $\mathrm{VCcCC}_{+20 \mathrm{mv}}: P=4.2 \times 10^{-19}$; Fig. $6 \mathrm{~B}$ and C). Also, the distribution clearly broadened, indicating a lower accuracy of the coupling of VS and V1 spikes (Fig. 6B). Overall, we found a clear positive correlation between the graded membrane potential and the VS-V1 latency (Fig. 6C, Pearson's correlation coefficient $r=0.94, P=0.005)$. The latency between pre- and postsynaptic spikes was also tested in a VS2/3-V1 pair. A significantly shorter latency compared with the bridged mode was present when the presynaptic VS2/3 was clamped in VCcCC to resting level or to a level hyperpolarized by $10 \mathrm{mV}$ (mean latencies $\pm \mathrm{SD}$ : $\mathrm{VCcCC}_{-10 \mathrm{mV}}: 0.600 \pm 0.894 \mathrm{~ms} ; \mathrm{VCcCC}_{\mathrm{RMP}}: 0.696 \pm 0.979 \mathrm{~ms}$; BM: $0.71 \pm 0.929 \mathrm{~ms}$; Student's $t$-test, BM vs. VCcCC: $P=0.004$; $\mathrm{BM}$ vs. $\mathrm{VCcCC}_{-10 \mathrm{mV}}: P=0.0006$; data not shown). In contrast, depolarization by $20 \mathrm{mV}$ during $\mathrm{VCcCC}$ led to a significantly larger latency between $\mathrm{VS} 2 / 3-\mathrm{V} 1$ spikes (mean latencies \pm $\mathrm{SD}: \mathrm{VCcCC}_{+20 \mathrm{mv}}$ : $0.860 \pm 0.944 \mathrm{~ms}$; Student's $t$-test: $\mathrm{BM}$ vs. $\left.\mathrm{VCcCC}_{+20 \mathrm{mv}}: P=0.002\right)$. Note that although the spike rates of the V1 neuron did not differ significantly between the control condition and voltage clamp of one presynaptic neuron in the VCcCC mode to resting level, we found significant differences in the VS-V1 latency between these conditions.

\section{Presynaptic spiking influences the pattern of postsynaptic spiking}

As a consequence of electrical coupling between VS neurons, synchronous spikes can be expected to occur not only in VS and V1 but also, at least to some extent, in neighbouring VS neurons. As was recently demonstrated, it is likely that synchronous spiking activity of VS neurons is responsible for the occurrence of spike doublets in V1 (Beckers et al., 2009). V1 spike doublets, which we characterize as a sequence of two spikes within $<4 \mathrm{~ms}$, frequently occur during recording in BM (Fig. 7A). The presence of V1 spike doublets becomes evident in the frequency distribution of interspike intervals (Fig. 7A-C). Beckers et al. (2009) have shown that the spike doublet rate dropped significantly when one of the VS neurons was voltage clamped to its resting level. A plausible explanation for this finding was that voltage clamping of one VS neuron interferes with spike synchronization between VS neurons. As a consequence, the probability of the postsynaptic V1 neuron receiving two inputs, each from one of the VS neurons within a brief time interval, is decreased. We now hypothesize that, if synchronization between VS neurons and the corresponding occurrence of spike doublets depends on the spike component of VS, it should persist when only the graded potential is set to resting level by $\mathrm{VCcCC}$. To test this hypothesis, the recording mode was alternated between BM, voltage clamp and $\mathrm{VCcCC}$ either to the resting membrane potential or to hyperpolarizing or depolarizing levels of 10 or $20 \mathrm{mV}$, respectively.

As shown in Fig. 4B and D, the V1 spike rates for the investigated conditions differed only moderately. Nevertheless, analysis of the interspike intervals revealed a change in the temporal distribution of the V1 spikes. When VS1 was voltage clamped to the resting membrane potential, V1 spike doublet rate dropped from $46.3 \%$ (confidence interval $45.2-47.3 \%$ ) in the unclamped situation to $37.2 \%$ (confidence interval $35.1-39.2 \%$, adjusted Wald method, the difference is significant when the $95 \%$ confidence intervals do not overlap; see Fig. 7), confirming the findings of Beckers et al. (2009). In contrast, clamping 

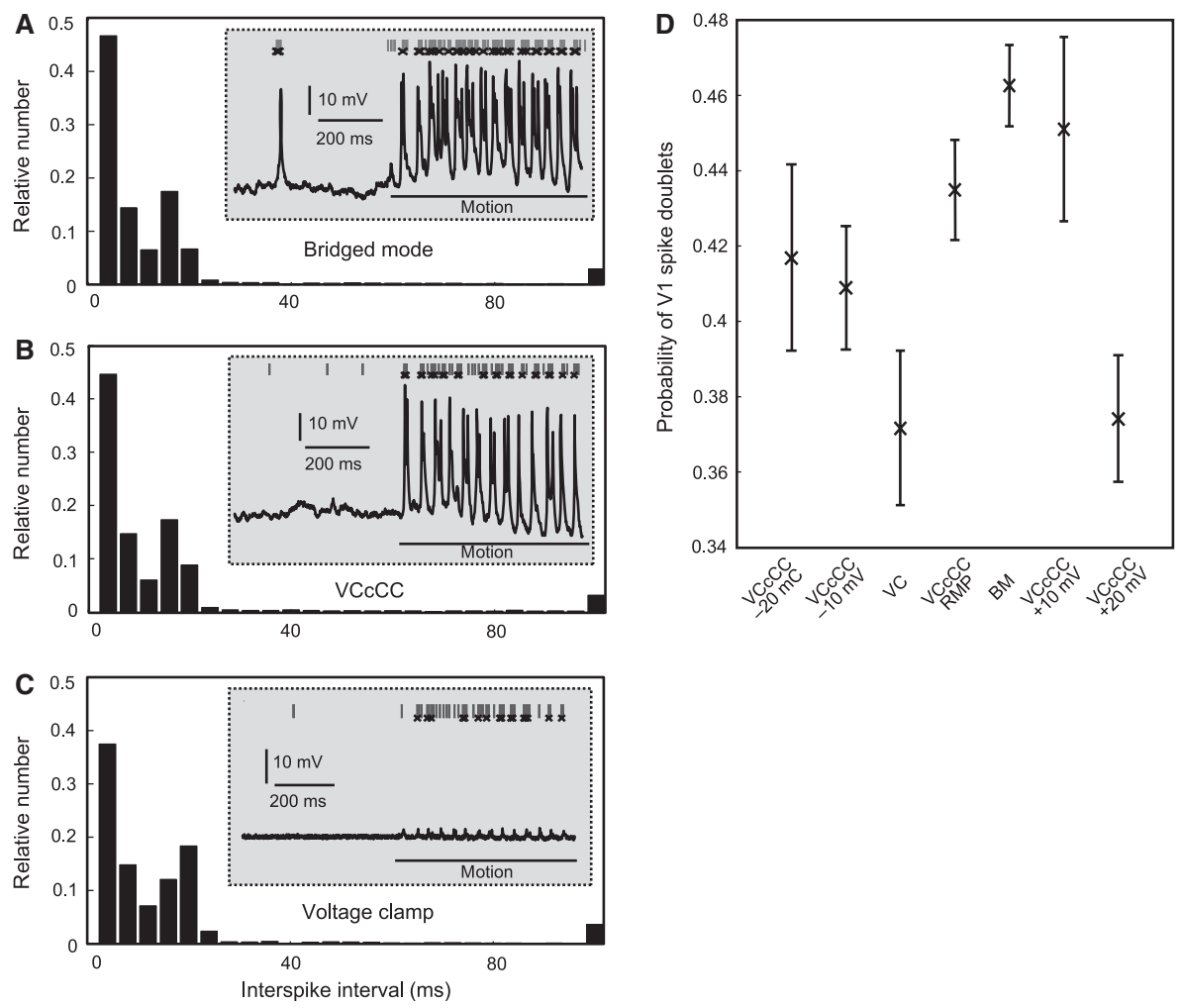

FIG. 7. Impact of voltage clamp and $\mathrm{VCcCC}$ of one VS neuron to its resting potential during presentation of motion in the preferred direction on the interspike interval distribution of $\mathrm{V} 1$. The histograms show the frequency distribution of the interval between two spikes (abscissa) in bridged mode (A), VCcCC (B), and full voltage clamp to resting potential at $-40 \mathrm{mV}(\mathrm{C})$. All three plots show the normalized data of the same neuron. Insets: example traces of presynaptic neuron membrane potential (bottom) and simultaneously registered postsynaptic spikes (top, dashes) for the different recording conditions. Doublet spikes (i.e. two postsynaptic spikes occurring within an interval of $<4 \mathrm{~ms}$ ) are marked by a cross. Data of one representative VS1-V1 neuron pair are shown. (D) V1 spike doublet probability calculated according to the adjusted Wald method (Wilson, 1927; Agresti \& Coull, 1998) in bridged mode, voltage clamp to the VS neuron's resting potential and $\mathrm{VCcCC}$ to either resting potential or to a hyper- or depolarized command potential, respectively. Error bars denote $95 \%$ confidence intervals. The differences in doublet rates are significant when these bars do not overlap. Total number of V1 spikes: $\mathrm{VCcCC}_{-20} \mathrm{mV}: 1476$; $\mathrm{VCcCC}_{-10 \mathrm{mV}}: 3319$; $\mathrm{VC}_{\mathrm{RMP}}$ : 2055 ; $\mathrm{VCcCC}_{\mathrm{RMP}}: 5058$; BM: 7873; $\mathrm{VCcCC}_{+10 \mathrm{mV}}: 1518 ; \mathrm{VCcCC}_{+20 \mathrm{mV}}: 3002$.

only the graded membrane potential component of VS1 in the VCcCC mode to its resting level resulted in a significantly weaker drop of the V1 doublet rate than under full voltage clamp (confidence intervals $42.2-44.2 \%$ vs. 35.1-39.2\%; cf. Fig. 7B and C). This finding corroborates our hypothesis that the occurrence of spike doublets in V1 depends on the spike component of the membrane potential of presynaptic VS neurons. Nevertheless, the decrease in the frequency of V1 spike doublets under VCcCC of VS1 to resting level just reached statistical significance, and hyperpolarizing $\mathrm{VCcCC}$ led to more pronounced drops in doublet rates (confidence intervals: $-10 \mathrm{mV}$ : 39.3-42.3\%; -20 mV: 39.2-44.2 \%; Fig. 7D). The latter finding might be a consequence of the diminished overall spike rate of V1 during presynaptic hyperpolarization. Surprisingly, a depolarization of VS1 by $20 \mathrm{mV}$ also led to a significant drop in the doublet rate (confidence interval 35.7-39.1\%; Fig. 7D). This effect might be a consequence of the reduced precision of VS-V1 coupling (see Fig. 6B).

\section{Discussion}

Sensory cells or local interneurons in visual systems might use graded changes in membrane potential for information propagation or a mixture of graded signals and action potentials. In this study we investigated the distinct roles of these two forms in synaptic transfer in the fly visual system. We conclude that both the level of the membrane potential of the presynaptic VS neurons as well as their spikes control the spiking activity of the postsynaptic V1 neuron. When during visual stimulation sustained membrane depolarization was blocked but spikes were preserved, the postsynaptic response was slightly reduced relative to the control condition where both components were present. Moreover, when the visually induced graded membrane potential was replaced by artificial de- or hyperpolarization, the postsynaptic response to motion was increased or decreased, respectively. A modulatory effect of graded membrane potential changes on spikemediated synaptic transmission has recently been demonstrated in neocortex, hippocampus and brainstem of mammals (Awatramani et al., 2005; Alle \& Geiger, 2006; Shu et al., 2006). However, transmission at the respective synapses differs from that examined in our study. Instead of only modulating the efficiency of spike-mediated transmission, the graded potential alone has been shown to be transmitted at fly VS-V1 synapses, in the absence of spikes (Beckers et al., 2007). Moreover, in the vertebrate model systems fast chemical synaptic signal transfer was investigated, whereas at VS-V1 synapses chemical and electrical signal transfer probably coexist (Haag \& Borst, 2008; Beckers et al., 2009).

\section{Methodological considerations concerning the use of VCcCC for the study of synaptic signal transfer}

The technique used in the present study, VCcCC (Sutor et al., 2003), allowed us to manipulate the presynaptic graded potential while 
presynaptic spikes could still be elicited by visual stimulation. Our study is, to our knowledge, the first using $\mathrm{VCcCC}$ to manipulate the responses of neurons to sensory stimuli for the investigation of synaptic transmission to their postsynaptic targets. Previously, $\mathrm{VCcCC}$ was used during sensory stimulation of spider mechanoreceptor neurons to test whether modulatory GABAergic inputs to the neurons exert their specific effects on firing rate and information coding via changes in conductance or changes in membrane potential (Pfeiffer \& French, 2009; Pfeiffer et al., 2009). VCcCC acts by incorporating low-pass filters with selectable time constants into the voltage clamp feedback circuit to control the amplifier's response speed. We therefore tested how different time constants affect the potential response of VS neurons to visual motion (Figs 2B and C, and 3). In their experiments Sutor et al. (2003) suggested setting the time constant to $\tau=100 \mathrm{~s}$ to monitor postsynaptic potentials and spikes at different background holding potentials. Such long time constants were inadequate for our aim to block graded potential changes soon after motion onset (data not shown). We decided to set the VCcCC time constant routinely to $0.5 \mathrm{~s}$ as a compromise between the ability for fast compensation of motion-induced graded potential responses and the degree of unwanted attenuation and distortion of membrane potential transients. We are aware that the VCcCC technique does not allow a perfect separation between graded potential and action potentials. However, for two reasons we believe that presynaptic spikes were not considerably affected by VCcCC. First, we systematically analysed the spikes (Fig. 2C) and did not find consistent effects of VCcCC on the spike width (data not shown). Second, attenuation of spikes as a side-effect of $\mathrm{VCcCC}$ is unlikely because spike amplitude increased when motion-induced graded depolarization was blocked. This observation is consistent with earlier reports about the effects current injections have on spike amplitude in VS neurons (Hengstenberg, 1977). A plausible explanation for the modulation of spike amplitude with the membrane potential level is that a certain fraction of the sodium channels show voltage-dependent tonic inactivation. Additionally, tonic hyperpolarization increases the driving force for sodium ions, facilitating the generation of large spikes.

As a complementary approach to VCcCC, sodium channel blockers might be used to selectively abolish the presynaptic spike component. Unfortunately, drugs that can be applied via the recording electrode would be required to restrict the pharmacological effect to a single neuron (Haag \& Borst, 1997). The more convenient bath application of a drug would affect the entire visual pathway and, in particular, block postsynaptic spiking activity.

\section{Regulation of V1 spike rate by the graded membrane potential and spiking activity of presynaptic VS neurons}

We examined how the activity of a postsynaptic neuron is controlled by the graded membrane potential and the spikes of one of its presynaptic neurons. In contrast to a former approach in which the presynaptic neuron was voltage-clamped to various holding potentials (Beckers et al., 2007) we used visual stimulation and applied VCcCC to manipulate the graded presynaptic potential component. The two approaches differ in one important respect. During voltage clamp of a presynaptic VS neuron the input of the postsynaptic V1 neuron is largely restricted to the clamped neuron. In contrast, visual stimulation activates VS1-VS3, because their receptive fields are very similar. Thus it is not surprising that the reduction in spike rate when blocking the graded signal of one of the VS neurons by VCcCC is fairly weak and variable. This reduction was typically in a range of $10 \mathrm{~Hz}$ for VS1. In contrast, voltage clamping VS1 to a similar tonic depolar- ization as during visual stimulation, $10 \mathrm{mV}$ relative to resting potential, already increased postsynaptic spike rate by about $30 \mathrm{~Hz}$ (Beckers et al., 2007).

In a previous study it was found that spikes elicited in a VS neuron by brief current pulses increase the probability that V1 fires a spike within a short latency (Beckers et al., 2009). Our approach in the present study enabled us to preserve the temporal structure of the presynaptic spike component as it occurs during motion stimulation. Our results corroborate the notion that presynaptic spikes, in addition to the graded component, affect the postsynaptic spike rate. Moreover, we found that presynaptic spikes, rather than being all-or-none signals, show during visual stimulation strong differences in their amplitude. These differences correlate with the level of the graded membrane potential and are relevant for their impact on the postsynaptic neuron - hyperpolarization is associated with larger amplitudes (see also Hengstenberg, 1977) and leads to shorter latencies between spikes in VS and V1. A similar effect of the membrane potential on spike amplitude was found in cricket auditory neurons (Baden \& Hedwig, 2010) and in pyramidal neurons of ferret prefrontal cortex and rat visual cortex (de Polavieja et al., 2005; Shu et al., 2006). In these cortical neurons the change in amplitude was inversely correlated with a change in spike width. This finding explains why Shu et al. (2006) found that the magnitude of the postsynaptic potentials, which were elicited by presynaptic spikes, increased with presynaptic depolarization. In contrast, at VS-V1 synapses the presynaptic depolarization level increases the latency between spikes in VS and V1. This result is consistent with our observation that in VS neurons, spike amplitude decreases with presynaptic depolarization, whereas spike width is not consistently affected. An alternative explanation for the different effects of presynaptic depolarization on postsynaptic responses is the different type of synaptic coupling. In Shu et al. (2006) chemical synapses were analysed, whereas at VS-V1 synapses most likely chemical and electrical coupling coexists (Haag \& Borst, 2008; Beckers et al., 2009). Thus, graded changes in membrane potential of VS neurons are directly transmitted to V1 and might change the $\mathrm{Na}^{+}$channel availability. A depolarization of the membrane potential might lead to an inactivation of $\mathrm{Na}^{+}$channels. This could result in longer latencies between spikes in VS and V1, because the inactivated $\mathrm{Na}^{+}$channels do no longer contribute to the spike generation in V1.

The enhancement of spike amplitude during presynaptic hyperpolarization of VS neurons and the associated decrease in the latency between spikes of VS and V1 might be functionally significant in a behavioural context. VS neurons hyperpolarize during motion in the non-preferred direction. Spikes would then be elicited by a sudden reversal of the motion direction, which is most likely linked to a rapid change in flight attitude, or when a moving object enters the receptive field. Such discontinuities in the stimulus would then elicit postsynaptic responses within a short latency and with a large probability (Beckers et al., 2009) and might thus be helpful to convey the relevant information to the neck motor system (Huston \& Krapp, 2008) and to descending neurons (Wertz et al., 2009) leading to reliable and quick motor responses. The interplay between graded changes in the membrane potential and spikes could thus form a mechanism for the context-dependent regulation of the reliability and the temporal precision of synaptic transmission. In another fly tangential neuron, the $\mathrm{H} 1$ neuron, temporal precision down to the sub-millisecond scale has been demonstrated (Nemenman et al., 2008). Interestingly, such precise timing was not a general feature of $\mathrm{H} 1$ spikes, but occurred in response to distinct events, such as abrupt transitions in motion velocity. 


\section{Dependency of temporal structure of postsynaptic spike responses on the presynaptic spike component}

In the V1 neuron small bursts of spikes, mostly occurring in groups of only two spikes (spike doublets), are considered to be the result of electrical coupling within the VS-V1 network and the resulting synchronous activity of two VS neurons (Beckers et al., 2009). The rate of spike doublets was reduced by voltage clamping one of the VS neurons to its resting potential (Beckers et al., 2009). In the present study we were able to show that the spike component of the presynaptic signal is important for the generation of spike doublets, because blocking only the graded component via $\mathrm{VCcCC}$ was nearly ineffective to reduce the rate of postsynaptic spike doublets. It has been shown in other invertebrate and vertebrate systems that long stretches of neural activity convey a different aspect of the sensory input than the presence of short bursts of spikes (Gabbiani et al., 1996; Marsat \& Pollack, 2006; Schwartz et al., 2007; Marsat et al., 2009). In a particular class of retinal ganglion cells, for example, bursts of spikes are triggered by a motion reversal (Schwartz et al., 2007). Spike doublets in V1 often occur directly after the cessation of motion in the non-preferred direction or at its reversal to motion in the preferred direction (see Fig. 2A), presumably resulting from synchronization between VS neurons and from the ability of large VS spikes to elicit temporally tightly coupled spikes in V1 (see Fig. 6). The use of more complex stimuli, for example naturalistic optic flow reconstructed after monitoring the trajectory and the gaze direction of flies during flight (Hateren \& Schilstra, 1999; Lindemann et al., 2003; Kern et al., 2005), may help to resolve whether spike doublets and other distinct patterns of spikes are associated with particular aspects of the visual input.

\section{Acknowledgements}

This work was supported by the Deutsche Forschungsgemeinschaft (DFG grant KU 1520/4-1). We thank M. Egelhaaf, U. Beckers, P. Hennig and two anonymous reviewers for their comments on earlier versions of the manuscript.

\section{Abbreviations}

$\mathrm{BM}$, bridged mode; RMP, resting membrane potential; $\mathrm{VCcCC}$, voltage clampcontrolled current clamp; VS neurons, neurons of the vertical system.

\section{References}

Agresti, A. \& Coull, B. (1998) Approximate is better than 'exact' for interval estimation of binomial proportions. Am. Stat., 52, 119-126.

Alle, H. \& Geiger, J.R. (2006) Combined analog and action potential coding in hippocampal mossy fibers. Science, 311, 1290-1293.

Alle, H. \& Geiger, J.R. (2008) Analog signalling in mammalian cortical axons. Curr. Opin. Neurobiol., 18, 314-320.

Awatramani, G.B., Price, G.D. \& Trussell, L.O. (2005) Modulation of transmitter release by presynaptic resting potential and background calcium levels. Neuron, 48, 109-121.

Baden, T. \& Hedwig, B. (2010) Primary afferent depolarization and frequency processing in auditory afferents. J. Neurosci., 30, 14862-14869.

Beckers, U., Egelhaaf, M. \& Kurtz, R. (2007) Synapses in the fly motion-vision pathway: evidence for a broad range of signal amplitudes and dynamics. J. Neurophysiol., 97, 2032-2041.

Beckers, U., Egelhaaf, M. \& Kurtz, R. (2009) Precise timing in fly motion vision is mediated by fast components of combined graded and spike signals. Neuroscience, 160, 639-650.

Borst, A. \& Egelhaaf, M. (1989) Principles of visual motion detection. Trends Neurosci., 12, 297-306.

Borst, A., Haag, J. \& Reiff, D.F. (2010) Fly motion vision. Annu. Rev. Neurosci., 33, 49-70.
Egelhaaf, M., Kern, R., Krapp, H.G., Kretzberg, J., Kurtz, R. \& Warzecha, A.K. (2002) Neural encoding of behaviourally relevant visual-motion information in the fly. Trends Neurosci., 25, 96-102.

Gabbiani, F., Metzner, W., Wessel, R. \& Koch, C. (1996) From stimulus encoding to feature extraction in weakly electric fish. Nature, 384, 564-567.

Haag, J. \& Borst, A. (1997) Encoding of visual motion information and reliability in spiking and graded potential neurons. J. Neurosci., 17, 48094819.

Haag, J. \& Borst, A. (1998) Active membrane properties and signal encoding in graded potential neurons. J. Neurosci., 18, 7972-7986.

Haag, J. \& Borst, A. (2004) Neural mechanism underlying complex receptive field properties of motion-sensitive interneurons. Nat. Neurosci., 7, 628-634.

Haag, J. \& Borst, A. (2008) Electrical coupling of lobula plate tangential cells to a heterolateral motion-sensitive neuron in the fly. J. Neurosci., 28, 1443514442.

Haag, J., Theunissen, F. \& Borst, A. (1997) The intrinsic electrophysiological characteristics of fly lobula plate tangential cells: II. Active membrane properties. J. Comput. Neurosci., 4, 349-369.

Hateren, J.H. \& Schilstra, C. (1999) Blowfly flight and optic flow. II. Head movements during flight. J. Exp. Biol., 202, 1491-1500.

Hausen, K. (1984) The lobula-complex of the fly: structure, function and significance in visual behaviour. In Ali, M.A. (Ed.), Photoreception and Vision in Invertebrates. Plenum Press, New York, pp. 523-559.

Hengstenberg, R. (1977) Spike responses of 'non-spiking' visual interneurone. Nature, 270, 338-340.

Hengstenberg, R., Hausen, K. \& Hengstenberg, B. (1982) The number and structure of giant vertical cells (VS) in the lobula plate of the blowfly Calliphora erythrocephala. J. Comp. Physiol. A., 149, 163-177.

Hooper, S.L. \& DiCaprio, R.A. (2004) Crustacean motor pattern generator networks. Neurosignals, 13, 50-69.

Huston, S.J. \& Krapp, H.G. (2008) Visuomotor transformation in the fly gaze stabilization system. PLoS Biol., 6, e173.

Juusola, M., French, A.S., Uusitalo, R.O. \& Weckström, M. (1996) Information processing by graded-potential transmission through tonically active synapses. Trends Neurosci., 19, 292-297.

Kalb, J., Egelhaaf, M. \& Kurtz, R. (2006) Robust integration of motion information in the fly visual system revealed by single cell photoablation. J. Neurosci., 26, 7898-7906.

Kern, R., van Hateren, J.H., Michaelis, C., Lindemann, J.P. \& Egelhaaf, M. (2005) Function of a fly motion-sensitive neuron matches eye movements during free flight. PLoS Biol., 3, e171.

Krapp, H.G., Hengstenberg, B. \& Hengstenberg, R. (1998) Dendritic structure and receptive-field organization of optic flow processing interneurons in the fly. J. Neurophysiol., 79, 1902-1917.

Krapp, H.G., Hengstenberg, R. \& Egelhaaf, M. (2001) Binocular contributions to optic flow processing in the fly visual system. J. Neurophysiol., 85, 724 734.

Kurtz, R., Warzecha, A.K. \& Egelhaaf, M. (2001) Transfer of visual motion information via graded synapses operates linearly in the natural activity range. J. Neurosci., 21, 6957-6966.

Lindemann, J.P., Kern, R., Michaelis, C., Meyer, P., van Hateren, J.H. \& Egelhaaf, M. (2003) FliMax, a novel stimulus device for panoramic and highspeed presentation of behaviourally generated optic flow. Vision Res. 43, 779-791.

Longden, K.D. \& Krapp, H.G. (2009) State-dependent performance of opticflow processing interneurons. J. Neurophysiol., 102, 3606-3618.

Maimon, G., Straw, A.D. \& Dickinson, M.H. (2010) Active flight increases the gain of visual motion processing in Drosophila. Nat. Neurosci., 13, 393-399.

Marder, E. \& Calabrese, R.L. (1996) Principles of rhythmic motor pattern generation. Physiol. Rev., 76, 687-717.

Marsat, G. \& Pollack, G.S. (2006) A behavioral role for feature detection by sensory bursts. J. Neurosci., 26, 10542-10547.

Marsat, G., Proville, R.D. \& Maler, L. (2009) Transient signals trigger synchronous bursts in an identified population of neurons. J. Neurophysiol., 102, 714-723

Nemenman, I., Lewen, G.D., Bialek, W. \& de Ruyter van Steveninck, R.R. (2008) Neural coding of natural stimuli: information at sub-millisecond resolution. PLoS Comput. Biol., 4, e1000025.

Pfeiffer, K. \& French, A.S. (2009) GABAergic excitation of spider mechanoreceptors increases information capacity by increasing entropy rather than decreasing jitter. J. Neurosci., 29, 10989-10994.

Pfeiffer, K., Panek, I., Höger, U., French, A.S. \& Torkkeli, P.H. (2009) Random stimulation of spider mechanosensory neurons reveals long-lasting excitation by GABA and muscimol. J. Neurophysiol., 101, 54-66. 
de Polavieja, G.G., Harsch, A., Kleppe, I., Robinson, H.P. \& Juusola, M. (2005) Stimulus history reliably shapes action potential waveforms of cortical neurons. J. Neurosci., 25, 5657-5665.

Rosner, R., Egelhaaf, M. \& Warzecha, A.K. (2010) Behavioural state affects motion-sensitive neurones in the fly visual system. J. Exp. Biol., 213, 331 338

de Ruyter van Steveninck, R.R. \& Laughlin, S.B. (1996) The rate of information transfer at graded-potential synapses. Nature, 379, 642-645.

Schwartz, G., Taylor, S., Fisher, C., Harris, R. \& Berry, M.J. (2007) Synchronized firing among retinal ganglion cells signals motion reversal. Neuron, 55, 958-969.

Selverston, A.I., Russell, D.F. \& Miller, J.P. (1976) The stomatogastric nervous system: structure and function of a small neural network. Prog. Neurobiol. 7, 215-290.
Shu, Y., Hasenstaub, A., Duque, A., Yu, Y. \& McCormick, D.A. (2006) Modulation of intracortical synaptic potentials by presynaptic somatic membrane potential. Nature, 441, 761-765.

Sutor, B., Grimm, C. \& Polder, H.R. (2003) Voltage-clamp-controlled currentclamp recordings from neurons: an electrophysiological technique enabling the detection of fast potential changes at preset holding potentials. Pflugers Arch., 446, 133-141.

Warzecha, A.K., Kurtz, R. \& Egelhaaf, M. (2003) Synaptic transfer of dynamic motion information between identified neurons in the visual system of the blowfly. Neuroscience, 119, 1103-1112.

Wertz, A., Haag, J. \& Borst, A. (2009) Local and global motion preferences in descending neurons of the fly. J. Comp. Physiol. A., 195, 1107-1120.

Wilson, E.B. (1927) Probable inference, the law of succession, and statistical inference. J. Am. Stat. Assoc., 22, 209-212. 